\title{
A continuum model for slip transfer at grain boundaries
}

\author{
Claude Fressengeas $^{1}$ and Manas V. Upadhyay ${ }^{2^{*}}$ (1)
}

\author{
${ }^{*}$ Correspondence: \\ manas.upadhyay@polytechnique.edu \\ ${ }^{2}$ Laboratoire de Mécanique des \\ Solides (LMS), CNRS, Ecole \\ Polytechnique, Institut \\ Polytechnique de Paris, 91128 \\ Palaiseau, France \\ Full list of author information is \\ available at the end of the article
}

\begin{abstract}
Using a continuous representation of dislocations in elastoplastic polycrystals, we investigate slip transfer at grain boundaries by assessing the compatibility of the slip system shear rates with tangential continuity of the plastic distortion rate tensor at these interfaces. Fulfillment of this tangential continuity condition is needed for consistency of the continuous description of dislocations in polycrystals. We show that, in f.c.c. materials at moderate temperatures, this condition unequivocally translates into constraints on the slip rates on both sides of grain boundaries. Appended to the elastoplastic boundary value problem, it allows a complete determination of the slip system shear rates. An algorithm enabling the implementation of compatible slip transfer in both the finite element methods and the spectral methods based on Fast Fourier Transforms is provided in both standard crystal plasticity and the mechanics of dislocations fields.
\end{abstract}

\section{Introduction}

Grain boundaries have a significant impact on plasticity mediated by dislocation glide. By acting as barriers to dislocation motion, they may limit the mean free path of dislocations and hinder plasticity. The Hall-Petch law [23,38], which describes the evolution of the yield stress as a function of the inverse square root of the average grain size, is a well-known manifestation of this limiting effect. Nevertheless, dislocations may be transferred across grain boundaries, either indirectly, because their accumulation in pile-ups on one side of the boundary may induce large internal stresses able to activate dislocation glide or even new dislocation sources on the other side, or directly because they may interact with the boundary, be absorbed and re-emitted on a different slip system, while leaving a residual part along the boundary. The present understanding of slip transmission across boundaries has been developed through numerous investigations of particular grain boundaries, from which several slip transfer criteria have been proposed [29,31$33,45]$. In these papers, different aspects of the phenomenon were invoked to predict the experimentally observed slip transfer reactions. [29] assumed tangential continuity of the plastic strain tensor field across the boundary and drew the consequence that at least four slip systems have to operate between the two crystals, distributed either with two in each crystal or with three in one and one in the other. However, as we shall see below, they did

(c) The Author(s) 2020. This article is licensed under a Creative Commons Attribution 4.0 International License, which permits use, sharing, adaptation, distribution and reproduction in any medium or format, as long as you give appropriate credit to the original author(s) and the source, provide a link to the Creative Commons licence, and indicate if changes were made. The images or other third party material in this article are included in the article's Creative Commons licence, unless indicated otherwise in a credit line to the material. If material is not included in the article's Creative Commons licence and your intended use is not permitted by statutory regulation or exceeds the permitted use, you will need to obtain permission directly from the copyright holder. To view a copy of this licence, visit http://creativecommons.org/licenses/by/4.0/. 
not recognize the slip incompatibility possibly arising from the tangential discontinuity of the plastic rotation field and failed to follow further that path. Instead, they proposed a criterion based on the resolved shear stress being the largest on the outgoing slip system. [45] combined two criteria: in the first one, the angle between the emission and pile-up slip planes should be minimized, and in the second one, the outgoing slip direction should maximize the resolved shear stress, as previously conjectured by [29]. [32] suggested that the residual dislocation left in the grain boundary plane also plays a role in slip transfer: not only the angle of the slip planes in both crystals, but also the slip directions angle should be minimized to promote slip transfer. Thus, Burgers vector conservation is involved in slip transfer. Burgers vector conservation and tangential continuity of the plastic distortion are closely related as will be recalled below, implying an intimate connection between the results in [32] and the context set forth in [29]. To predict which outgoing slip system is favorable, [33] proposed a cumulative set of criteria closely related to the above ones, namely: (1) the angle between the lines of intersection of the incoming and outgoing slip planes with the boundary should be as small as possible; (2) the resolved shear stress acting on the possible outgoing slip systems should be as large as possible and (3) the magnitude of the Burgers vector of the residual dislocations left at the grain boundary should be a minimum. Since then, these criteria have been qualitatively confirmed by experimental observations in various materials $[5,6,24,28]$.

Predominantly, the above slip transfer criteria are motivated by slip system and grain boundary geometry as well as the state of internal stress within a continuum framework. They do not resolve atomic level interactions between dislocations and grain boundaries. An additional level of analysis is made possible by simulations at atomic scale, e.g. using molecular dynamics (MD) simulations. Atomistic simulations provide approaches where the grain boundary core structure can be thoroughly described at a resolution length scale of the order of inter-atomic spacing $[10,18,48]$. The goal of such simulations in the field of slip transfer has been to explore the atomic interactions over a wide range of metallic materials and grain boundary structures in order to improve the accuracy of slip transfer prediction criteria (see for example [12-14] and the review in [44]). However, from the possible five degrees of freedom of a grain boundary, an infinite space of possible slip transfer configurations exists, while only a relatively small amount has been analyzed with MD simulations. A more complete survey of the pertinent variables is needed to probe more completely the space of slip transmission events, which clearly represents an overwhelming challenge to the MD community. Therefore, continuum models built either by embedding the three main slip transfer criteria indicated above, or by proposing model mechanisms for grain boundary and dislocation interactions are of great interest in complement to atomistic simulations. They are able to encompass all these situations, and may in addition allow tackling large polycrystalline samples submitted to realistic loading. Common to all continuum crystal plasticity models solving boundary value problems is the incorporation of the resolved shear stresses due to the inherent modeling of plastic slip, which can be either combined with an introduction of the geometric transfer criteria, or with a continuous model for the description of grain boundaries and dislocations [8].

Models aimed at describing slip transfer across grain boundaries from a continuum perspective include surface-dislocation density based approaches, non-exhaustively represented here by reference [21]. In these models, surface-dislocation densities are defects designed to accommodate tangential discontinuities of the plastic distortion [7,19]. Their 
support is limited to the infinitely thin interface itself. Such a description may be taken more or less literally and accepted in certain circumstances: electron microscopy has revealed that the structure of low angle boundaries or semi-coherent interfaces actually involves dislocations $[11,36]$. In most cases however, the so-called surface-dislocations cannot be identified with observable dislocations and reduce to being only mathematical artefacts. For example, they cannot represent the actual structure of high angle boundaries, because their spacing would have to be so small that their cores would overlap [30,39]. As a result, surface-dislocation-based modeling approaches fail to account for the structure and energy of high-angle boundaries, because they overlook their core properties. In addition, by allowing the accommodation of any tangential discontinuity of the elastic/plastic distortion and distortion rate, they tend to reduce grain-to-grain interactions, even for low-angle boundaries, with consequences on the prediction of texture evolution [34], size and Bauschinger effects $[37,43,46]$ and plastic strain localization [47]. Here we similarly foresee consequences on slip transfer.

In the present work, we also have in mind a mesoscale representation where the spatial resolution length scale is not sufficiently small to reveal the core structure of the grain boundaries, but allows instead encompassing large polycrystals at relatively low cost. Indeed, we partly see grain boundaries as interfaces of vanishingly small thickness, across which the stress, total distortion, elastic/plastic distortion and distortion rate components may experience a discontinuity. However, following [2,20], we contend that all such discontinuities are not acceptable and that some continuity is mandatory for consistency of a field approach to dislocations. Besides tangential continuity of the total distortion/distortion rate tensor-the so-called Hadamard compatibility conditions [22], which ensures continuity of the body across the interface, and normal continuity of the stress tensor (the traction vector needs to be continuous across the interface to respect mechanical equilibrium), we require tangential continuity of the plastic distortion/distortion rate tensors across the interface, thus following the steps taken in the early work of [29] on slip transfer. Such a statement induces non-locality of the elasto-plastic response of the polycrystal across the interface, because elastic/plastic distortion values from the left of the interface have to be equal to their counterparts from the right. It amounts to viewing the dislocations that accumulate at grain boundaries as a continuous density field defined over a finite boundary layer, perhaps of a small thickness-but definitely not vanishingly small. Such non-locality was shown to have a strong impact on the elastic/plastic strain and rotation fields, both in the vicinity of grain boundaries and throughout the body. As already suggested above, it allowed retrieving such complex features as size and morphology effects, loading path-dependency, the Bauschinger effect and directional hardening in the plastic response of particle-reinforced alloys and thin polycrystalline fims $[37,43,46]$, overall texture intensity and a $\beta$ fiber more consistent with experimental observation in f.c.c. metals than the Taylor models [34], and shear strain localization in lamellar Al-Cu-Li alloys that conventional crystal plasticity fails to capture [47].

In the present paper, slip transfer at grain boundaries, from grain to grain at the level of each slip system, is described on the basis of Burgers vector conservation and tangential continuity of the plastic distortion rate at interfaces. The model is grounded in the mechanical theory of continuously distributed dislocations [1,26,27], but it may also be applied to crystal plasticity appended with tangential continuity of the plastic distortion rate. The outline of the paper is therefore as follows. After setting up notations in "Nota- 
tions" section, a primer for the mechanics of dislocation fields is presented in "Mechanics of dislocation fields" section and tangential continuity conditions on the plastic/total distortion/distortion rate tensors across interfaces are reviewed in "Tangential continuity constraints along interfaces" section. "Slip transfer" section presents the application to slip transfer. In "Numerical procedures" section, numerical procedures are proposed for the implementation of the model in both standard crystal plasticity and the mechanics of dislocation fields. Conclusions on the potential applicability of the analysis to the plasticity of polycrystals follow.

\section{Notations}

A bold symbol denotes a tensor, as in: A. When there may be ambiguity, an arrow is superposed to represent a vector: $\overrightarrow{\mathbf{V}}$. The transpose of tensor $\mathbf{A}$ is $\mathbf{A}^{t}$. All tensor subscript indices are written with respect to the basis $\left(\mathbf{e}_{i}, i=1,2,3\right)$ of a rectangular Cartesian coordinate system. Vertical arrays of one or two dots represent contraction of the respective number of "adjacent" indices on two immediately neighboring tensors, in standard fashion. For example, the tensor A.B with components $A_{i k} B_{k j}$ results from the dot product of tensors $\mathbf{A}$ and $\mathbf{B}$, and $\mathbf{A}: \mathbf{B}=A_{i j} B_{i j}$ represents their inner product. The cross product of a second order tensor $\mathbf{A}$ and a vector $\mathbf{V}$, the div and curl operations for second order tensors are defined row by row, in analogy with the vectorial case. For example:

$$
\begin{aligned}
(\mathbf{A} \times \mathbf{V})_{i j} & =e_{j k l} A_{i k} V_{l} \\
(\operatorname{div} \mathbf{A})_{i} & =A_{i j, j} \\
(\operatorname{curl} \mathbf{A})_{i j} & =e_{j k l} A_{i l, k} .
\end{aligned}
$$

where $e_{j k l}=\mathbf{e}_{j} .\left(\mathbf{e}_{k} \times \mathbf{e}_{l}\right)$ is a component of the third-order alternating Levi-Civita tensor $\mathbf{X}$, equal to 1 if the $j k l$ permutation is even, -1 if it is odd and 0 otherwise. In the component representation, the comma followed by a component index indicates a spatial derivative with respect to the corresponding Cartesian coordinate as in relations (2,3). A vector $\overrightarrow{\mathbf{A}}$ is associated with tensor $\mathbf{A}$ by using the inner product of $\mathbf{A}$ with tensor $\mathbf{X}$ :

$$
\begin{aligned}
& (\overrightarrow{\mathbf{A}})_{k}=-\frac{1}{2}(\mathbf{X}: \mathbf{A})_{k}=-\frac{1}{2} e_{k i j} A_{i j} \\
& (\mathbf{A})_{i j}=-(\mathbf{X} \cdot \overrightarrow{\mathbf{A}})_{i j}=-e_{i j k}(\overrightarrow{\mathbf{A}})_{k} .
\end{aligned}
$$

The symmetric and skew-symmetric parts of tensor $\mathbf{A}$ are denoted $\mathbf{A}^{\text {sym }}$ and $\mathbf{A}^{\text {skew }}$ respectively. Given a unit vector $\mathbf{n}$ normal to an interface $I$ in a domain $D$ and orienting $I$ from sub-domain $D^{-}$to sub-domain $D^{+}$, the normal part $\mathbf{A}_{n}$ and tangential part $\mathbf{A}_{t}$ of tensor A are

$$
\begin{aligned}
& \mathbf{A}_{n}=\mathbf{A} \cdot \mathbf{n} \otimes \mathbf{n} \\
& \mathbf{A}_{t}=\mathbf{A}-\mathbf{A}_{n}=\mathbf{A} \cdot(\mathbf{I}-\mathbf{n} \otimes \mathbf{n}),
\end{aligned}
$$

where $\mathbf{I}-\mathbf{n} \otimes \mathbf{n}$ is an operator performing tangential projection. For a vector $\mathbf{V}$ :

$$
\begin{aligned}
& \mathbf{V}_{n}=(\mathbf{V} \cdot \mathbf{n}) \mathbf{n}=V_{n} \mathbf{n} \\
& \mathbf{V}_{t}=\mathbf{V}-\mathbf{V}_{n} .
\end{aligned}
$$

The discontinuity of a tensor $\mathbf{A}$ at the interface $I$ is denoted $\llbracket \mathbf{A} \rrbracket=\mathbf{A}^{+}-\mathbf{A}^{-}$, where $\mathbf{A}^{-}$and $\mathbf{A}^{+}$are the limits of tensor $\mathbf{A}$ when evaluated at limit points on the interface along direction $\mathbf{n}$ in $D^{-}$and $D^{+}$, respectively. A superposed dot represents a material time derivative. 


\section{Mechanics of dislocation fields}

In the present framework, continuity of the displacement vector field $\mathbf{u}$ along with continuity of its derivatives, is assumed at any point in a simply-connected body undergoing elasto-plastic deformation, except perhaps along interfaces where continuity of the derivatives may not hold. Consequently, the distortion tensor can be defined as the gradient of the displacement $\mathbf{U}=\operatorname{grad} \mathbf{u}$. As such, it is curl-free:

$$
\operatorname{curl} \mathrm{U}=0 .
$$

Equation (10) is a necessary condition for the integrability of the displacement $\mathbf{u}$ and a compatibility condition for the distortion $\mathbf{U}$. However, in the presence of dislocations, the elastic, $\mathbf{U}_{e}$, and plastic, $\mathbf{U}_{p}$, components of $\mathbf{U}$ are incompatible. Indeed, if dislocations thread a patch $S$ in the body, a constant discontinuity $\mathbf{b}$ in the elastic displacement exists across $S$, and manifests itself as a closure defect along the circuit $C$ surrounding $S$ :

$$
\mathbf{b}=\llbracket \mathbf{u}_{e} \rrbracket=\int_{C} \mathbf{U}_{e} \cdot \mathbf{d} \mathbf{l} .
$$

$C$ is referred to as a Burgers circuit and $\mathbf{b}$ as the Burgers vector of this dislocation ensemble. By virtue of Stoke's theorem:

$$
\int_{C} \mathbf{U}_{e} \cdot \mathbf{d l}=\int_{S} \operatorname{curl} \mathbf{U}_{e} \cdot \mathbf{n} d S,
$$

the discontinuity $\mathbf{b}$ can be characterized in a pointwise continuous manner by the tensor $\alpha$ :

$$
\operatorname{curl} \mathbf{U}_{e}=\alpha
$$

such that

$$
\mathbf{b}=\int_{S} \boldsymbol{\alpha} \cdot \mathbf{n} d S .
$$

$\boldsymbol{\alpha}$ is known as Nye's dislocation density tensor [35]. In its presence, there is an incompatible (non curl-free) part, $\mathbf{U}_{e}^{\perp}$, of the elastic distortion tensor. By invoking the Stokes-Helmholtz decomposition of a square-integrable tensor field with square-integrable first order derivatives [25], it is possible to find uniquely the square-integrable tensor and vector fields $\phi$ and $\mathbf{z}$ such that the elastic distortion field $\mathbf{U}_{e}$ reads as the sum:

$$
\mathbf{U}_{e}=\operatorname{curl} \phi+\operatorname{grad} \mathbf{z}
$$

Taking the curl of $\mathbf{U}_{e}$ in Eq. (15) extracts $\operatorname{curl} \phi$ and discards $\operatorname{grad} \mathbf{z}$, whereas taking its divergence extracts $\operatorname{grad} \mathbf{z}$ and eliminates curl $\phi$. Therefore, Eq. (13) actually involves only $\operatorname{curl} \phi$, which we identify as the incompatible part $\mathbf{U}_{e}^{\perp}$ of $\mathbf{U}_{e}$ :

$$
\operatorname{curl} \mathrm{U}_{e}^{\perp}=\operatorname{curl} \operatorname{curl} \phi=\alpha .
$$

In addition, $\mathbf{g r a d} \mathbf{z}$ can be identified as the compatible part $\mathbf{U}_{e}^{\|}$of the elastic distortion $\mathbf{U}_{e}$, while $\mathbf{z}$ is the compatible elastic displacement $\mathbf{u}_{e}^{\|}$, up to a constant. However, Eq. (16) is still insufficient to determine $\mathbf{U}_{e}^{\perp}$ from a given dislocation field $\boldsymbol{\alpha}$. To ensure correctness of this 
identification, $\mathbf{U}_{e}^{\perp}$ must vanish identically throughout the body when $\boldsymbol{\alpha}=0$. Following $[4,25]$, we therefore augment Eq. (16) with the side conditions

$$
\operatorname{div} \mathbf{U}_{e}^{\perp}=0 \quad \text { and } \quad \mathbf{U}_{e}^{\perp} \cdot \mathbf{n}=0 \text {, }
$$

the latter being imposed on the external boundary $\partial \mathcal{B}$ with unit normal $\mathbf{n}$, to ensure that its solution does not contain a gradient part. Taking the curl of Eq. (16), we then find:

$$
\operatorname{curl} \operatorname{curl} \mathbf{U}_{e}^{\perp}=\operatorname{grad} \operatorname{div} \mathbf{U}_{e}^{\perp}-\operatorname{div} \operatorname{grad} \mathbf{U}_{e}^{\perp}=\operatorname{curl} \alpha,
$$

and therefore, using the side conditions (17):

$$
\operatorname{div} \operatorname{grad} \mathbf{U}_{e}^{\perp}=-\operatorname{curl} \boldsymbol{\alpha}, \mathbf{U}_{e}^{\perp} \cdot \mathbf{n}=0 \quad \text { on } \partial \mathcal{B} .
$$

Equation (19) is a Poisson equation for the unknown $\mathbf{U}_{e}^{\perp}$ whose solution under the above boundary condition vanishes identically throughout the body when $\alpha=0$, as required. Similarly, we could have evidenced the existence of an incompatible part of the plastic distortion, $\mathbf{U}_{p}^{\perp}$, opposite to the incompatible elastic distortion $\mathbf{U}_{e}^{\perp}$ and such that lattice continuity is maintained. Further, a curl-free compatible component, $\mathbf{U}_{p}^{\|}$of the plastic distortion $\mathbf{U}_{p}$, may also exist, as well as a compatible plastic displacement $\mathbf{u}_{p}^{\|}$. The following relations are therefore satisfied:

$$
\begin{aligned}
\mathbf{U} & =\mathbf{U}_{e}+\mathbf{U}_{p} \\
\mathbf{U}_{e} & =\mathbf{U}_{e}^{\perp}+\mathbf{U}_{e}^{\|} \\
\mathbf{U}_{p} & =\mathbf{U}_{p}^{\perp}+\mathbf{U}_{p}^{\|} \\
0 & =\mathbf{U}_{e}^{\perp}+\mathbf{U}_{p}^{\perp}
\end{aligned}
$$

if infinitesimal transformations are assumed. Using Eqs. $(16,23)$, it is readily seen that the relation

$$
\boldsymbol{\alpha}=-\operatorname{curl} \mathbf{U}_{p}^{\perp}
$$

is equivalent to Eq. (16), and that similarly the equation

$$
\boldsymbol{\alpha}=-\operatorname{curl} \mathbf{U}_{p}
$$

is also valid. Note that the continuity condition:

$$
\operatorname{div} \alpha=0
$$

follows directly from Eqs. $(13,16)$.

The kinematics of dislocation densities derives from the conservation of the Burgers vector during their motion across arbitrary material patches. Consider a material surface $S$ bounded by a closed curve $C$. Let $\mathbf{f}$ be the dislocation flux field used to measure the rate of inflow into $S$ of dislocation lines, carrying along with them their corresponding Burgers vectors through a line element $\mathbf{d x}$ of curve $C$. Let $\mathbf{V}$ be the velocity of the dislocations with respect to the lattice. In the absence of dislocation sources, the conservation of the 
Burgers' vector content demands that the rate of change of the Burgers' vector of all dislocation lines threading $S$ be equal to the total dislocation flux across curve $C$ :

$$
\frac{d}{d t} \int_{S} \boldsymbol{\alpha} . \mathbf{n} d S=\int_{C} \mathbf{f} . \mathbf{d x} .
$$

Due to Stokes' theorem, the point-wise statement corresponding to (27) is, for small transformations:

$$
\dot{\alpha}=\text { curl f }
$$

where $\dot{\alpha}$ represents the time derivative of the dislocation density tensor. As shown in [3]:

$$
\mathbf{f}=-\boldsymbol{\alpha} \times \mathbf{V}
$$

Consequently, the local statement of balance (28) becomes:

$$
\dot{\alpha}+\operatorname{curl} \quad(\alpha \times \mathbf{V})=0 .
$$

Equation (30) is referred to as a transport law for the dislocation density tensor $\boldsymbol{\alpha}$. It can be understood as an evolution equation for $\boldsymbol{\alpha}$ when the dislocation velocity $\mathbf{V}$ is provided as a function of the stress state and dislocation character through constitutive statements. With this information, Eq. (30) constitutes a natural basis for the dynamic description of dislocation microstructures. Its meaning is that, through the curl term, the incompatible part of the dislocation flux incrementally feeds the dislocation density. Comparing Eqs. $(25,30)$ it follows, after time derivation of Eq. (25), that the cross product $\boldsymbol{\alpha} \times \mathbf{V}$ can be identified with the plastic distortion rate tensor $\dot{\mathbf{U}}_{p}$, up to a gradient:

$$
\begin{aligned}
\dot{\alpha}+\operatorname{curl} \dot{\mathbf{U}}_{p} & =0 \\
\dot{\mathbf{U}}_{p} & =\boldsymbol{\alpha} \times \mathbf{V}+\operatorname{grad} \dot{\mathbf{u}}_{p}^{*} .
\end{aligned}
$$

At microscale, there is no physical mechanism that could be described by the term grad $\dot{\mathbf{u}}_{p}^{*}$, which has therefore to be cancelled. Equation (32) then describes plasticity solely from $\boldsymbol{\alpha}$ dislocation motion. At mesoscale $\operatorname{grad} \dot{\mathbf{u}}_{p}^{*}$ can be given the significance of a statistical plastic distortion rate, meaning that plasticity may be obtained even when the dislocation density tensor vanishes at this scale. Indeed, using space-time running averages of the dislocation density tensor $\boldsymbol{\alpha}$, dislocation velocity $\mathbf{V}$ and plastic distortion rate tensor $\dot{\mathbf{U}}_{p}$ over a domain of mesoscopic size, allows writing the mesoscopic plastic distortion rate $\overline{\dot{\mathbf{U}}_{p}}$ as:

$$
\overline{\mathbf{U}_{p}}=\overline{\boldsymbol{\alpha} \times \mathbf{V}}=\overline{\boldsymbol{\alpha}} \times \overline{\mathbf{V}}+\mathbf{L}_{p},
$$

where overbars indicate averaged variables [4]. It is seen that $\overline{\mathbf{U}_{p}}$ may be non-zero when the net dislocation density vanishes at mesoscale $(\overline{\boldsymbol{\alpha}}=0)$, in which case it becomes:

$$
\overline{\mathbf{U}_{p}}=\mathbf{L}_{p}
$$


and is to be physically interpreted as the distortion rate produced by the so-called "statistical dislocations". Dropping the overbars for convenience, the plastic distortion rate may be written at this scale as:

$$
\dot{\mathbf{U}}_{p}=\boldsymbol{\alpha} \times \mathbf{V}+\mathbf{L}_{p} .
$$

Averaging in space thus provides a link with conventional crystal plasticity: $\mathbf{L}_{p}$ may be derived from well-established slip system-based constitutive relationships for the viscoplasticity of crystalline materials. At microscale, the relation $\dot{\mathbf{U}}_{p}=\boldsymbol{\alpha} \times \mathbf{V}$ may be seen as a tensorial Orowan relationship in the continuum, not making reference to the cristallography and slip systems of the material. However, we look here for a microscale expression of $\dot{\mathbf{U}}_{p}$, such that $\mathbf{L}_{p}=0$ and $\mathbf{V} \neq 0$, but with such an explicit reference to cristallography through the incoming and outgoing slip systems at grain boundaries. This is different from the averaged point of view of [34] who assumed instead $\mathbf{L}_{p} \neq 0$ and $\mathbf{V}=0$. Consider f.c.c. materials: on the one hand, they feature four $<111>$ slip planes with unit normal vectors $\mathbf{m}_{s}$, each with three [11̄0] slip directions $\mathbf{s}_{s}$, and therefore twelve slip systems with Schmid tensors $\mathbf{P}_{s}=\mathbf{s}_{s} \otimes \mathbf{m}_{s}, s \in(1,12)$. In each slip plane, only two out of three slip directions are independent, and therefore only eight slip systems out of twelve are independent. On the other hand, $\dot{\mathbf{U}}_{p}$ has eight independent components if pressure independence of plasticity is assumed. In such conditions, the relation

$$
\dot{\mathbf{U}}_{p}=\sum_{s \in(1,8)} \dot{\gamma}_{s} \mathbf{P}_{s}
$$

between the components of the plastic distortion rate $\dot{\mathbf{U}}_{p}$ and the shear rates $\dot{\gamma}_{s}$ on the independent slip systems $\mathbf{P}_{s}, s \in(1,8)$ is a full rank linear algebraic system of eight equations for the eight unknowns $\dot{\gamma}_{s}$, allowing to describe uniquely plasticity mediated by dislocation glide in terms of slip system shear rates [43]. Assuming pressure independence implies that Eq. (36) does not account for dislocation climb. However, it still accounts for cross-slip. In b.c.c. and h.c.p. materials, the number of independent slip systems is material-dependent, and it may be as high as 48 in $\alpha-F e$ in b.c.c. materials and twelve in h.c.p. materials, thereby obviously precluding any such one-to-one correspondence.

In a small pertubation setting, the rest of the equations of the mechanics of dislocation fields is not different from standard crystal plasticity, to which the theory reduces when the dislocation density tensor is formally set to zero. Assuming linear elasticity, the stress tensor $\mathbf{T}$ is obtained from the tensor $\mathbf{C}$ of elastic moduli and elastic strain tensor $\boldsymbol{\epsilon}_{e}=\mathbf{U}_{e}^{\text {sym }}$ as

$$
\mathbf{T}=\mathbf{C}: \boldsymbol{\epsilon}_{e}
$$

Neglecting inertial forces and any volumetric force density as unessential for the present purposes, it satisfies the balance of momentum equation

$$
\operatorname{div} \mathbf{T}=0
$$

Complemented with constitutive relations for the dislocation velocity $\mathbf{V}$ as a function of the stress and dislocation density tensors and for the shear strain rates $\dot{\gamma}_{s}$ involved in the 
plastic velocity gradient $\mathbf{L}_{p}$ as a function of the stress tensor, Eqs. $(19,20-23,31,35,37,38)$ form a complete set of equations, of hyperbolic character, for the evolution of the displacement and dislocation density fields. Boundary conditions comprise the conventional stress and displacement conditions.

\section{Tangential continuity constraints along interfaces}

Material properties and/or field variables, such as the elastic/plastic displacement and distortion/distortion rate fields or the dislocation density field may encounter discontinuities across surfaces such as grain boundaries in polycrystals. However, as indicated above, not all discontinuities are admissible in the present framework: mechanical balance, the continuity of matter and the conservation of the Burgers vector across such interfaces mandate satisfaction of partial continuity conditions. To recall these conditions, we assume the existence of a surface of discontinuity $I$ separating the body $\mathcal{B}$ into two sub-domains $\mathcal{B}^{-}$ and $\mathcal{B}^{+}$. At any point $\mathrm{P}$ on $I$, the unit normal vector $\mathbf{n}$ to the interface is oriented from $\mathcal{B}^{-}$toward $\mathcal{B}^{+}$, and we denote by $\mathbf{l}$ and $\boldsymbol{\tau}=\mathbf{n} \times \mathbf{l}$ two unit vectors belonging to the interface (see Fig. 1). In the absence of cracks and shocks, continuum mechanics respectively requires that the displacement $\mathbf{u}$ and traction vector $\mathbf{t}=$ T.n be continuous across the interface: $\llbracket \mathbf{u} \rrbracket=0, \llbracket \mathbf{t} \rrbracket=0$. The continuity of the traction vector is reflected as well by the continuity of the normal part, $\mathbf{T}_{n}=\mathbf{T} . \mathbf{n} \otimes \mathbf{n}$, of the stress tensor: $\llbracket \mathbf{T}_{n} \rrbracket=0$, whereas the tangential part $\mathbf{T}_{t}=\mathbf{T}-\mathbf{T}_{n}$ of the latter may be discontinuous across the interface. Continuity of the displacement at the interface requires that the total distortion $\mathbf{U}$ be a gradient tensor and therefore satisfy

$$
\int_{C} \mathbf{U} \cdot \mathbf{d x}=0
$$

along the rectangular closed circuit $C$ lying across the interface as shown in Fig. 1 . When $C$ is collapsed onto point $P$ by letting $L \rightarrow 0, h^{-} \rightarrow 0, h^{+} \rightarrow 0$, the limit of the above integral provides the interfacial relation

$$
\forall \mathbf{l} \in I, \quad \llbracket \mathbf{U} \rrbracket . \mathbf{l}=0
$$

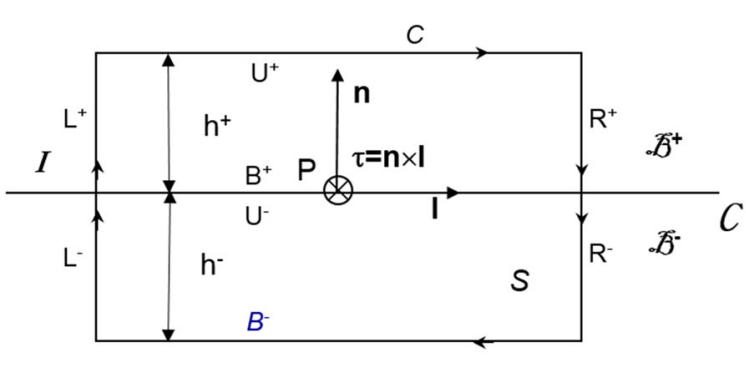

Fig. 1 Burgers circuit $C=U^{+} \cup R^{+} \cup R^{-} \cup B^{-} \cup L^{-} \cup L^{+}$across an interface / separating the body $\mathcal{B}$ into domains $\mathcal{B}^{-}, \mathcal{B}^{+} . \mathbf{l}$ is the unit tangent to curve $\mathcal{C}, \mathbf{n}$ is the unit normal to interface $l$, and $\boldsymbol{\tau}=\mathbf{n} \times \mathbf{l}$ the "tangent normal" to the bounded surface $S$ and curve $\mathcal{C}$ 
whose meaning is tangential continuity of the total distortion. This property is also rendered more compactly as:

$$
\llbracket \mathbf{U} \rrbracket \times \mathbf{n}=0,
$$

or as

$$
\llbracket \mathbf{U}_{t} \rrbracket=\llbracket \mathbf{U}-\mathbf{U} . \mathbf{n} \otimes \mathbf{n} \rrbracket=0 .
$$

The discontinuity of the distortion is therefore limited to its normal part $\llbracket \mathbf{U}_{n} \rrbracket$. Eqs. $(41,42)$ are known as Hadamard's compatibility conditions [22]. By differentiating Eq. (39) with respect to time, tangential continuity is also found to apply to the total distortion rate $\dot{\mathbf{U}}$ :

$$
\begin{array}{r}
\forall \mathbf{l} \in I, \quad \llbracket \dot{\mathbf{U}} \rrbracket . \mathbf{l}=0 \\
\llbracket \dot{\mathbf{U}} \rrbracket \times \mathbf{n}=0,
\end{array}
$$

whereas the normal part $\dot{\mathbf{U}}_{n}$ of the distortion rate tensor may encounter a discontinuity. Of course, the satisfaction of relations $(41,42,44)$ precludes any occurrence of matter disruption mechanisms at interfaces, such as grain boundary sliding or cavitation. However, Hadamard's compatibility equations $(41,42)$ do not impose any constraint on the plastic distortion tensor $\mathbf{U}_{p}$ at the interface. We show below that tangential continuity conditions on $\mathbf{U}_{p}$ arise at the interface if the choice is made to represent continuously the dislocations in the interface area by adopting a small resolution length scale [2].

With this aim, we consider again the rectangular closed circuit $C$ lying across the interface in the manner shown in Fig. 1. The intersection of surface $S$ and interface $I$ defines a curve $\mathcal{C}$ on $I$, to which the orthonormal frame $\mathcal{D}=\left(P, \mathbf{e}_{1}=\mathbf{l}, \mathbf{e}_{2}=\boldsymbol{\tau}, \mathbf{e}_{3}=\mathbf{n}\right)$ is a natural frame at point $P$. A bulk areal dislocation density field $\boldsymbol{\alpha}$ is assumed to take place over surface $S$, and a surface-dislocation density $\boldsymbol{\alpha}^{S}(I)$ is provisionally allowed to exist along the interface $I$. To first comment on the role of the surface-dislocation density $\boldsymbol{\alpha}^{S}(I)$, we consider the limit of the Burgers vector content of circuit $C$

$$
\mathbf{b}=-\int_{C} \mathbf{U}_{p} \cdot \mathbf{l} d s=\int_{S} \boldsymbol{\alpha} \cdot \boldsymbol{\tau} d S
$$

when $C$ is collapsed onto point $P$ by letting $L \rightarrow 0, h^{-} \rightarrow 0, h^{+} \rightarrow 0$ as previously. We find:

$$
\forall \mathbf{l} \in I, \quad-\llbracket \mathbf{U}_{p} \rrbracket . \mathbf{l}=\boldsymbol{\alpha}^{S}(I) . \tau .
$$

This relation is nothing else than the celebrated Frank's relation of the theory of dislocations $[7,19]$, which provides

$$
\boldsymbol{\alpha}^{S}(I)=\llbracket \mathbf{U}_{p} \rrbracket \times \mathbf{n} .
$$

Its meaning is that, whatever the tangential discontinuity $\llbracket \mathbf{U}_{p} \rrbracket$ of the plastic distortion, it can be accommodated by an appropriate surface-dislocation density tensor $\boldsymbol{\alpha}^{S}(I)$. Note that $\boldsymbol{\alpha}^{S}(I)$ is referred to as the "grain boundary Burgers tensor" in [21]. It must be borne in mind that $\boldsymbol{\alpha}^{S}(I)$ and the dislocation density tensor $\boldsymbol{\alpha}$ are mathematical objects of a different nature. Whereas $\boldsymbol{\alpha}$ (expressed in units of Burgers vector length per unit surface) 
is a continuously defined field representing a volumetric dislocation ensemble in the bulk of the material, $\boldsymbol{\alpha}^{S}(I)$ (in units of Burgers vector length per unit length, i.e. non dimensional) is a surfacic density field supported by the singular interface $I$. As indicated in the introduction Section, these surface-dislocations cannot be identified with observable dislocations. They usually reduce to being only mathematical artefacts whose role is, as already stated, to accommodate a tangential discontinuity of the plastic distortion across the interface. In order to restore tangential continuity of the plastic distortion, we shall instead assume $\boldsymbol{\alpha}^{S}(I)=0$ in the following, in sharp contrast with Gurtin's model. Thus, we set the condition

$$
\forall \mathbf{l} \in I, \quad \llbracket \mathbf{U}_{p} \rrbracket . \mathbf{l}=0 .
$$

A more compact way of stating this property is alternatively:

$$
\llbracket \mathbf{U}_{p} \rrbracket \times \mathbf{n}=0,
$$

or, using the tangential part $\llbracket \mathbf{U}_{p, t} \rrbracket$ of $\llbracket \mathbf{U}_{p} \rrbracket$ :

$$
\llbracket \mathbf{U}_{p, t} \rrbracket=\llbracket \mathbf{U}_{p}-\mathbf{U}_{p} \cdot \mathbf{n} \otimes \mathbf{n} \rrbracket=0 .
$$

Equations $(48,49,50)$ are regularity conditions for the computation of the dislocation density tensor $\boldsymbol{\alpha}$ through Eq. (25). Only when they are satisfied can a continuous dislocation density tensor be defined across the interface [17]. As already suggested, the effect of such a constraint is to distribute smoothly the dislocation density arising from plastic distortion incompatibility at the interface over a finite width volumetric boundary layer. In doing so, nonlocal interactions between domains $\mathcal{B}^{-}$and $\mathcal{B}^{+}$across the interface are enhanced, because values of the plastic distortion at limit points on either sides of the interface have to be equal. Of course, the interface conditions $(48,49,50)$ do not put constraints on the normal discontinuity $\llbracket \mathbf{U}_{p, n} \rrbracket=\llbracket \mathbf{U}_{p} \cdot \mathbf{n} \otimes \mathbf{n} \rrbracket$, which generally involves a plastic shear jump with components in the local frame $\mathcal{D}\left(\llbracket \epsilon_{13}^{p} \rrbracket=\llbracket \epsilon_{31}^{p} \rrbracket, \llbracket \epsilon_{23}^{p} \rrbracket=\llbracket \epsilon_{32}^{p} \rrbracket\right)$, a tilt rotation jump $\left(\llbracket \omega_{1}^{p} \rrbracket, \llbracket \omega_{2}^{p} \rrbracket\right)$ and a normal stretch jump $\llbracket \epsilon_{33}^{p} \rrbracket$ in pressure-sensitive materials. However, compatibility conditions between these normal discontinuities arise when several interfaces with respective discontinuities of the plastic distortions $\llbracket \mathbf{U}_{p} \rrbracket_{i}, i \in(1,2 \ldots, N)$ connect along a multiple-line, in practice a triple-line with $N=3$ in polycrystals. Indeed, closure requires that the sum of all discontinuities vanish at the multiple-line:

$$
\sum_{i=1}^{N} \llbracket \mathbf{U}_{p} \rrbracket_{i}=0
$$

because the same grain is used to start and finish a closed circuit around the multiple-line. Summing the relations (49) for all interfaces, and using Eq. (51), it is seen that the normal discontinuities in the plastic distortion need to satisfy a Kirchhoff-type relation at the multiple-line:

$$
\sum_{i=1}^{N} \llbracket \mathbf{U}_{p} \rrbracket_{i} \cdot \mathbf{n}_{i} \otimes \mathbf{n}_{i}=0
$$


When all normals $\mathbf{n}_{i}$ are coplanar, Eq. (52) reduces to an Herring-type relationship at the multiple-line [20].

Now, performing the time-derivative of the net Burgers vector obtained from the circuit $C$ in Eq. 45, collapsing $C$ onto point $P \in I$ by letting again $L \rightarrow 0, h^{-} \rightarrow 0, h^{+} \rightarrow 0$, we obtain the jump condition

$$
\forall \mathbf{l} \in I, \quad \llbracket \dot{\mathbf{U}}_{p} \rrbracket . \mathbf{l}=0,
$$

which reflects both Burgers vector conservation at the interface and tangential continuity of $\dot{\mathbf{U}}_{p}$ [2]. Equation (53) may alternatively read in compact form:

$$
\llbracket \dot{\mathbf{U}}_{p} \rrbracket \times \mathbf{n}=0
$$

or equivalently

$$
\llbracket \dot{\mathbf{U}}_{p, t} \rrbracket=\llbracket \dot{\mathbf{U}}_{p}-\dot{\mathbf{U}}_{p} \cdot \mathbf{n} \otimes \mathbf{n} \rrbracket=0,
$$

or else:

$$
\dot{\mathbf{U}}_{p, t}^{-}=\dot{\mathbf{U}}_{p, t}^{+}
$$

Since the interface conditions $(54,55,56)$ ensure consistency of a field description of dislocations in a polycrystalline body, they must be appended to the boundary value problem in the mechanics of dislocation fields. They may also be appended to standard crystal plasticity, in which case the latter is augmented into a nonlocal model accounting for grain interactions [47].

\section{Slip transfer}

The tangential plastic distortion rate tensor has only six independent components. Therefore, six independent slip systems are required to enforce its continuity across an interface. This is in contrast with the four slip systems needed in [29] to fulfill tangential continuity of the plastic strain rate tensor, i.e. the symmetric part of the plastic distortion rate tensor. As already suggested, this last requirement overlooks the slip incompatibility possibly arising from the tangential discontinuity of the plastic rotation rate field, i.e. the skew-symmetric part of the plastic distortion rate tensor. Using Eqs. $(36,55)$, tangential continuity of the plastic distortion rate may read in f.c.c. materials:

$$
\llbracket \sum_{i \in(1,8)} \dot{\gamma}_{s} \mathbf{P}_{s} \cdot(\mathbf{I}-\mathbf{n} \otimes \mathbf{n}) \rrbracket=0 .
$$

We insist again that, in contrast with [34], Eq. (57) is not to be understood as an averaged equation. It represents a full rank algebraic linear system of six independent equations for the eight unknown shear rate jumps $\llbracket \dot{\gamma}_{s} \rrbracket$. Using Eq. (36), the projection $\dot{\gamma}$ of the plastic distortion rate tensor $\dot{\mathbf{U}}_{p}$ on any slip system with Schmid tensor $\mathbf{P}=\mathbf{s} \otimes \mathbf{m}$ is

$$
\dot{\gamma}=\dot{\mathbf{U}}_{p}: \mathbf{P}=\sum_{i \in(1,8)} \dot{\gamma}_{s}\left(\mathbf{s}_{s} \otimes \mathbf{m}_{s}\right):(\mathbf{s} \otimes \mathbf{m})=\sum_{i \in(1,8)} \dot{\gamma}_{s}\left(\mathbf{s}_{s} \cdot \mathbf{s}\right)\left(\mathbf{m}_{s} \cdot \mathbf{m}\right) .
$$


Note from this relation that for multislip, $\dot{\gamma}$ is generally different from the shear rate on this particular slip system. It may be decomposed into its tangential part, $\dot{\gamma}_{t}=\dot{\mathbf{U}}_{p, t}: \mathbf{P}$, and normal part, $\dot{\gamma}_{n}=\dot{\mathbf{U}}_{p, n}: \mathbf{P}$. We are concerned here only with the tangential part $\dot{\gamma}_{t}$, as the normal part $\dot{\gamma}_{n}$ is left unaffected by Eqs. (54-57). On any incoming slip system with Schmid tensor $\mathbf{P}^{-}=\mathbf{s}^{-} \otimes \mathbf{m}^{-}$, the tangential part of the projection is

$$
\dot{\gamma}_{t}^{-}=\dot{\mathbf{U}}_{p, t}^{-}: \mathbf{P}^{-}
$$

and on any outgoing slip system with Schmid tensor $\mathbf{P}^{+}=\mathbf{s}^{+} \otimes \mathbf{m}^{+}$:

$$
\dot{\gamma}_{t}^{+}=\dot{\mathbf{U}}_{p, t}^{+}: \mathbf{P}^{+}
$$

Substracting Eq. (59) from Eq. (60), we find

$$
\llbracket \dot{\gamma}_{t} \rrbracket=\dot{\mathbf{U}}_{p, t}^{+}: \mathbf{P}^{+}-\dot{\mathbf{U}}_{p, t}^{-}: \mathbf{P}^{-}=\llbracket \dot{\mathbf{U}}_{p, t}: \mathbf{P} \rrbracket,
$$

which is also, by decomposing the jump of the product:

$$
\llbracket \dot{\gamma}_{t} \rrbracket=\dot{\mathbf{U}}_{p, t}^{+}: \mathbf{P}^{+}-\dot{\mathbf{U}}_{p, t}^{-}: \mathbf{P}^{+}+\dot{\mathbf{U}}_{p, t}^{-}: \mathbf{P}^{+}-\dot{\mathbf{U}}_{p, t}^{-}: \mathbf{P}^{-}=\llbracket \dot{\mathbf{U}}_{p, t} \rrbracket: \mathbf{P}^{+}+\dot{\mathbf{U}}_{p, t}^{-}: \llbracket \mathbf{P} \rrbracket,
$$

and finally, using Eqs. $(55,56)$ :

$$
\llbracket \dot{\gamma}_{t} \rrbracket=\dot{\mathbf{U}}_{p, t}^{-}: \llbracket \mathbf{P} \rrbracket=\dot{\mathbf{U}}_{p, t}^{+}: \llbracket \mathbf{P} \rrbracket .
$$

Equation (63) is a relation constraining slip transfer from any incoming slip system $\mathbf{P}^{-}$ to any outgoing slip system $\mathbf{P}^{+}$. Once the tangential part of, say, the incoming plastic distortion rate is known, the slip discontinuity $\llbracket \dot{\gamma}_{t} \rrbracket$ is found from its contracted product with the jump of the Schmid tensor. In full detail, the left hand side relation in Eq. (63) is also

$$
\llbracket \dot{\gamma}_{t} \rrbracket=\left(\dot{\mathbf{U}}_{p}-\dot{\mathbf{U}}_{p} \cdot \mathbf{n} \otimes \mathbf{n}\right)^{-}: \llbracket \mathbf{P} \rrbracket=\sum_{i \in(1,8)} \dot{\gamma}_{s}^{-} \mathbf{s}_{s}^{-} \otimes\left(\mathbf{m}_{s}^{-}-\left(\mathbf{m}_{s}^{-} \cdot \mathbf{n}\right) \mathbf{n}\right): \llbracket \mathbf{s} \otimes \mathbf{m} \rrbracket,
$$

and

$$
\llbracket \dot{\gamma}_{t} \rrbracket=\sum_{i \in(1,8)} \dot{\gamma}_{s}^{-} \llbracket\left(\mathbf{s}_{s}^{-} \cdot \mathbf{s}\right)\left(\left(\mathbf{m}_{s}^{-}-\left(\mathbf{m}_{s}^{-} \cdot \mathbf{n}\right) \mathbf{n}\right) \cdot \mathbf{m}\right) \rrbracket=\sum_{i \in(1,8)} \dot{\gamma}_{s}^{-} \llbracket\left(\mathbf{s}_{s}^{-} \cdot \mathbf{s}\right)\left(\mathbf{m}_{s, t}^{-} \cdot \mathbf{m}\right) \rrbracket
$$

where $\mathbf{m}_{s, t}^{-}=\mathbf{m}_{s}^{-}-\left(\mathbf{m}_{s}^{-} \cdot \mathbf{n}\right) \mathbf{n}$ is the tangential part of $\mathbf{m}_{s}^{-}$. We observe from Eq. (63) that the same is true for the outgoing side of the interface:

$$
\llbracket \dot{\gamma}_{t} \rrbracket=\sum_{i \in(1,8)} \dot{\gamma}_{s}^{+} \llbracket\left(\mathbf{s}_{s}^{+} \cdot \mathbf{s}\right)\left(\mathbf{m}_{s, t}^{+} \cdot \mathbf{m}\right) \rrbracket
$$

and note that, in terms of the slip plane orientation and slip direction jumps, respectively $\llbracket \mathbf{m} \rrbracket$ and $\llbracket \mathbf{s} \rrbracket$, another expression for Eq. (65) is

$$
\llbracket \dot{\gamma}_{t} \rrbracket=\sum_{i \in(1,8)} \dot{\gamma}_{s}^{-}\left(\left(\mathbf{s}_{s}^{-} \cdot \mathbf{s}^{-}\right)\left(\mathbf{m}_{s, t}^{-} \cdot \llbracket \mathbf{m} \rrbracket\right)+\left(\mathbf{s}_{s}^{-} \cdot \llbracket \mathbf{s} \rrbracket\right)\left(\mathbf{m}_{s, t}^{-} \cdot \mathbf{m}^{-}\right)+\left(\mathbf{s}_{s}^{-} \cdot \llbracket \mathbf{s} \rrbracket\right)\left(\mathbf{m}_{s, t}^{-} \cdot \llbracket \mathbf{m} \rrbracket\right)\right) .
$$


The slip discontinuity $\llbracket \dot{\gamma}_{t} \rrbracket$ is therefore a quasi-linear function of the slip plane orientation and slip direction jumps, with a nonlinear term in $(\llbracket \mathbf{s} \rrbracket, \llbracket \mathbf{m} \rrbracket)$ suggesting that the criteria on slip plane orientation and slip direction are generally not independent of one another. Equation (67) is qualitatively consistent with the geometric criteria proposed by [31$33,45]$, but it provides a much more detailed quantitative description of slip transfer.

We now examine several simple examples to probe further Eqs. $(65,66,67)$ :

1. Assume first that the incoming and outgoing slip planes are parallel, with slip plane normals in the same direction: $\llbracket \mathbf{m} \rrbracket=0$. Then, according to Eq. (67),

$$
\llbracket \dot{\gamma}_{t} \rrbracket=\sum_{i \in(1,8)} \dot{\gamma}_{s}^{-}\left(\mathbf{s}_{s}^{-} \cdot \llbracket \mathbf{s} \rrbracket\right)\left(\mathbf{m}_{s, t}^{-} \cdot \mathbf{m}^{-}\right) .
$$

If in addition the slip direction is unchanged: $\llbracket \mathbf{s} \rrbracket=0$, then $\llbracket \dot{\gamma}_{t} \rrbracket=0$, meaning that there is no change in the tangential slip rate on this slip system across the boundary. Thus, dislocations can slip across the boundary as if it were invisible and, for single slip, the shear rate on this slip system is unchanged, as could be expected. If both slip planes are parallel to the interface: $\mathbf{m}_{s, t}^{-}=0$, then $\llbracket \dot{\gamma}_{t} \rrbracket=0$, meaning again that the tangential slip rate does not change across the boundary, but now whatever the slip directions. Instead, assume that both slip planes are normal to the interface: $\mathbf{m}_{s, t}^{-} \cdot \mathbf{m}^{-}=1$. Then slip transfer depends only on the jumps $\llbracket \mathbf{s} \rrbracket$ of the slip direction and orientation angles $\phi_{s}=\left(\mathbf{s}, \mathbf{s}_{s}\right): \llbracket \dot{\gamma}_{t} \rrbracket=\sum_{i \in(1,8)} \dot{\gamma}_{s}^{-}\left(\mathbf{s}_{s}^{-} \cdot \llbracket \mathbf{s} \rrbracket\right)=$ $\sum_{i \in(1,8)} \dot{\gamma}_{s}^{-} \llbracket \cos \phi_{s} \rrbracket$. Finally, assume that both slip planes are inclined at angle $\theta$ with the interface: $\mathbf{m}_{s, t}^{-} \cdot \mathbf{m}^{-}=\cos \theta$. Then the slip transfer varies smoothly with $\theta$ as $\llbracket \dot{\gamma}_{t} \rrbracket=\cos \theta \sum_{i \in(1,8)} \dot{\gamma}_{s}^{-} \llbracket \cos \phi_{s} \rrbracket$ between the last two configurations.

2. Assume now that the probed slip directions are orthogonal: $\mathbf{s}^{-} \cdot \mathbf{s}^{+}=0$. Then, Eq. (65) provides

$$
\llbracket \dot{\gamma}_{t} \rrbracket=-\sum_{i \in(1,8)} \dot{\gamma}_{s}^{-}\left(\mathbf{s}_{s}^{-} \cdot \mathbf{s}^{-}\right)\left(\mathbf{m}_{s, t}^{-} \cdot \mathbf{m}^{-}\right)=-\cos \theta \sum_{i \in(1,8)} \dot{\gamma}_{s}^{-}\left(\mathbf{s}_{s}^{-} \cdot \mathbf{s}^{-}\right),
$$

showing that slip is hindered at the interface. The extreme situation is obtained in the presence of single slip: $\mathbf{s}^{-}=\mathbf{s}_{s}^{-}$when the incoming slip plane is normal to the interface: $\cos \theta=1$. Then the slip discontinuity is maximized: $\llbracket \dot{\gamma}_{t} \rrbracket=-\dot{\gamma}_{s}^{-}$, meaning that slip is fully blocked at the interface, as could be expected.

We now assume that a non-trivial solution to the geometrical constraints (57) has been found, by using some yet unknown method, at all points of all interfaces in the body, such that all tangential jumps $\llbracket \dot{\gamma}_{t} \rrbracket$ satisfy the transfer relations (67) and all shear rate jumps $\left(\llbracket \dot{\gamma}_{s} \rrbracket, s \in(1,8)\right)$ satisfy the tangential continuity conditions (57). Any set of shear rate jumps $\left(\llbracket \lambda \dot{\gamma}_{s} \rrbracket, s \in(1,8), \lambda \in \mathbb{R}\right)$ proportional to this first solution also leads to a solution of Eq. (57). These $\lambda \mathrm{s}$ can in fact be seen as stress-dependent coefficients, and the actual $\lambda$ value should allow satisfying the balance of momentum equations and boundary conditions for the whole body. Thus, the geometric constraints (57) only provide a set of admissible shear rates, among which the actual values are obtained by using the stress field derived from the solution of the mechanical boundary value problem. Hence, the latter actually consists in the field equations for the unknown variables, subjected to standard initial and boundary conditions, and complemented with the interfacial constraints (57). 
Such a complexity of the problem may not have always been recognized in the literature devoted to slip transfer.

\section{Numerical procedures}

In this Section, we are interested in designing an approximate numerical method to determine slip transfer across grain boundaries based on the developments in "Slip transfer" section. The method should be sufficiently versatile to allow its implementation in both a finite element framework and a spectral FFT-based method. Previous work on the account of tangential continuity of the plastic distortion rate include the finite element simulations of $[37,40,41,43,50]$ and $[46,47]$. In a Galerkin scheme for the solution of Eq. (30), tangential continuity of the plastic distortion rate is naturally obtained in a weak sense from the choice of continuous test functions. In nodal finite element implementations, continuity of the compatible/incompatible elastic/plastic displacements is further assumed at all nodes along element interfaces, which implies tangential continuity of the corresponding elastic/plastic distortions, exactly at nodes and approximately between nodes. [37] uses additional multipoint constraints in a Galerkin-Least-Squares formulation [40,50]. Element interfaces coincide with grain boundaries and double nodes are defined along the boundaries with a different number on either side of the boundary. The involved fields are defined at each node and constrained to be equal at the double nodes, which allows modeling both the free flow of dislocations across the boundary and a completely blocked flow on either side of the boundary. Here, we follow the work of $[46,47]$ where the surfaces of discontinuity do not necessarily coincide with element interfaces and do not necessarily contain nodes. This is of interest in spectral FFT-based methods where plain rectangular grids are used, or in a finite element framework when possibly transgranular surfaces of discontinuity form during loading, as in shear banding [47]. We start with a solution of the elastoplastic boundary value problem in the polycrystalline body, not accounting for tangential continuity of the plastic distortion rate at grain boundaries. As a result, surfacedislocation densities are present along boundaries to accommodate the plastic distortion rate incompatibilities that have been arising between grains. We first manage to convert these surface-dislocations into a volumetric dislocation density distribution in the neighboring regions, then remove this distribution from the dislocation density field together with the corresponding incompatible plastic distortion rate (see details below). Updates of the dislocation density and incompatible plastic distortion rate fields are then needed throughout the body, and consequently the elasto-plastic solution of the boundary value problem also needs to be updated. Tangential continuity of the plastic distortion rate and slip transfer relations may then be checked at interfaces, and the procedure iterated until convergence if some residual discontinuity is found. The solutions provided in [47] in the context of augmented crystal plasticity show that, at convergence, tangential continuity of the plastic distortion rate is satisfied at grain boundaries. Further, transgranular shear band localization occurs that conventional crystal plasticity fails to predict. In [46], the solutions provided in the context of the mechanics of dislocation fields show that consequences of tangential continuity include the occurrence of dislocation pile-ups at grain boundaries, the activation of slip across boundaries, sample and grain size effects on mechanical behavior and induced anisotropy of hardening, i.e. the Bauschinger effect. As discussed in [47], the dislocation density and internal stress fields are very likely underes- 
timated in augmented crystal plasticity. In the mechanics of dislocation fields, tangential continuity of the plastic distortion rate ensures continuity of the dislocation density field at interfaces, and it is likely that a more complete account of internal stresses and slip transfer is reached. The foreseeable consequences on slip transfer were not thoroughly examined in these papers.

We now provide details on the enforcement of tangential continuity of the plastic distortion rate and surface-dislocation removal. Interfaces possibly cross mesh elements in finite element methods and certainly cross grids in FFT-based spectral methods. In the corresponding "boundary elements", nodes (or voxels in FFT) pertain to the grain on the left of the interface, others to the grain on the right, but they usually do not belong to the interface. Since all variables are actually known from their nodal values, it seems reasonable to consider the grain boundaries as spreading in practice over the closest nodes in these boundary elements. Hence, the surface-dislocation density satisfying the interfacial relation (54) also spreads over nodes in this area, and finding $\dot{\boldsymbol{\alpha}}^{S}(I)$ is numerically equivalent to incrementing a volumetric dislocation density $\Delta \boldsymbol{\alpha}^{I}$ through Eq. (31). In other words, the geometrically necessary dislocation density field $\Delta \boldsymbol{\alpha}^{I}$ identified at nodes on both sides of the interface reflects the interfacial density $\dot{\boldsymbol{\alpha}}^{S}(I)$ spread out over the same nodes. Thus, removing the surface-dislocation density $\dot{\alpha}^{S}(I)$ from the interface, is also numerically equivalent to removing the volumetric dislocation density $\Delta \boldsymbol{\alpha}^{I}$ from its close neighborhood. The benefit brought by this surfacic-to-volumetric conversion is that it allows substracting the corresponding incompatible plastic distortion rate field $\dot{\mathbf{U}}_{p}^{I, \perp}$ from the existing approximation of the plastic distortion rate field in the body. Thus, the following steps should be implemented at each time increment $\Delta t$ of an explicit code:

1. Compute the plastic distortion rate tensor $\dot{\mathbf{U}}_{p}$ using the stress field obtained from the solution of the elastic-plastic problem at the previous time step and update the plastic distortion tensor $\mathbf{U}_{p}$ in the body,

2. compute the increment $\Delta \boldsymbol{\alpha}=\dot{\boldsymbol{\alpha}} \Delta t$ in the body through Eq. (31),

3. select the nodes surrounding the interfaces and define the increment $\Delta \boldsymbol{\alpha}_{I}=\Delta \boldsymbol{\alpha}$ at these nodes, $\Delta \boldsymbol{\alpha}_{I}=0$ elsewhere,

4. update $\boldsymbol{\alpha}$ in the whole body using the increment $\Delta \boldsymbol{\alpha}-\Delta \boldsymbol{\alpha}_{I}$ (implying that $\Delta \boldsymbol{\alpha}$ is set to zero at the selected boundary nodes),

5. compute the incompatible plastic distortion $\mathbf{U}_{p}^{\perp, I}$ associated with the increment $\Delta \boldsymbol{\alpha}_{I}$ by solving the corresponding Poisson equation (19),

6. substract $\mathbf{U}_{p}^{\perp, I}$ from $\mathbf{U}_{p}$ in the whole body,

7. update the stress field by solving again the elasto-plastic boundary value problem,

8. update the $\dot{\mathbf{U}}_{p}$ field, check for tangential continuity and slip transfer,

9. go to step (2) if residual discontinuity.

The steps $(5,6,7)$ suggest that tangential continuity may have a long-range impact in the body, because removing the $\mathbf{U}_{p}^{\perp, I}$ field modifies the plastic distortion and stress fields in the entire body. In contrast, incrementing $\alpha$ through steps $(1,2)$ and ignoring steps (3-7), as commonly practiced in standard crystal plasticity simulations to provide an evaluation of the dislocation density field, yields an $\boldsymbol{\alpha}$-field localized in the vicinity of the interfaces and associated with undue tangential discontinuity of the plastic distortion rate. 


\section{Concluding remarks}

The present analysis of slip transfer is made in the generic context of a field theory of dislocations $[1,2,27]$. We posit Burgers vector conservation, which implies tangential continuity of the plastic distortion rate at grain boundaries, serving in turn as a basis for slip transfer predictions. In addition to being reasonable from a physical point of view, this fundamental postulate warrants smoothness of the dislocation density tensor field across interfaces and therefore consistency of the present continuous approach. The predictions are qualitatively consistent with the commonly accepted criteria for slip transfer [31-33,45], which adds confidence in the Burgers vector conservation postulate. Moreover, they complement these criteria with a detailed quantitative content based on slip system and interface geometry. However the shear rates on the slip systems involved in Burgers vector conservation/tangential continuity of the plastic distortion rate are stress dependent, which implies that slip transfer at grain boundaries does not depend only on interfacial conditions, but also on the solution of the complete elasto-plastic boundary value problem.

The present model for slip transfer is consistent with the existence of a continuous dislocation density tensor field throughout the body, including at surfaces of discontinuity, because the latter are required to involve only normal discontinuities of the plastic distortion and distortion rate. In contrast, models where the grain boundaries are seen as singular interfaces supporting surface-dislocations are inconsistent with a bulk description featuring a volumetric dislocation density tensor field because continuity of the latter is not verified at interfaces. The present slip transfer analysis is also consistent with crystal plasticity appended with tangential continuity of the plastic distortion rate, and it may be implemented in this context. Crystal plasticity augmented in this manner has nonlocal character through grains interacting with their neighbors, and the slip transfer relationships reflect these nonlocal interactions. However, the internal stresses associated with dislocation pile-ups at grain boundaries are likely to be more correctly estimated in the mechanics of dislocation fields $[1,2]$ than in this augmented version of crystal plasticity, because the incompatible elastic strains associated with the presence of dislocations are not accounted for in the latter, with predictable consequences on slip system activity on both sides of the interfaces.

In the present paper, the slip transfer model is restricted to pressure-insensitive f.c.c. materials. Dislocation climb is ruled out to preserve pressure independence of the plastic behavior, which implies moderate temperatures. However, out-of-plane motion of dislocations through cross-slip of screws is permitted. The model may be extended to specific b.c.c. and h.c.p. materials provided they feature $8 / 9$ independent slip systems. To take advantage of the gains in computing costs obtained from the spectral FFT-based methods [42], further work will be devoted to the implementation of tangential continuity of the plastic distortion rate and slip transfer relationships in the spectral methods recently developed for the numerical solution of periodic boundary value problems in the mechanics of dislocation fields $[9,15,16,49]$. Simulations of relatively large polycrystalline samples will be undertaken, either in augmented crystal plasticity or in the mechanics of dislocation fields, to investigate at once numerous grain boundary/slip system configurations while keeping computation costs acceptable. 


\section{Acknowledgements}

The authors thank an anonymous reviewer for drawing their attention to the natural account of tangential continuity in the weak sense in Galerkin methods.

\section{Authors' contributions}

CF and MVU designed the model. Both authors contributed to writing the manuscript, CF being the main writer. Both authors read and approved the final manuscript.

\section{Funding}

CF gratefully acknowledges financial support from the institute INSIS of CNRS and University of Lorraine.

\section{Availability of data and materials}

Not applicable.

\section{Competing interests}

The authors declare that they have no competing interests.

\section{Author details}

'Laboratoire d'Etude des Microstructures et de Mécanique des Matériaux LEM3, Université de Lorraine/CNRS/Arts et Métiers ParisTech, 7 rue Félix Savart, 57070 Metz, France, ${ }^{2}$ Laboratoire de Mécanique des Solides (LMS), CNRS, Ecole Polytechnique, Institut Polytechnique de Paris, 91128 Palaiseau, France.

Received: 3 November 2019 Accepted: 6 February 2020

Published online: 10 March 2020

\section{References}

1. Acharya A. A model of crystal plasticity based on the theory of continuously distributed dislocations. J Mech Phys Solids. 2001:49:761-84.

2. Acharya A. Jump condition for GND evolution: a connection between grain growth and slip transmission at grain boundaries. Philos Mag. 2007;87:1349-59.

3. Acharya A. Microcanonical entropy and mesoscale dislocation mechanics and plasticity. J Elast. 2011;104:23-44.

4. Acharya A, Roy A. Size effects and idealized dislocation microstructure at small scales: predictions of a phenomenological model of Mesoscopic Field Dislocation Mechanics: part I. J Mech Phys Solids. 2006;54:1687-710.

5. Bieler TR, Alizadeh R, Peña Ortega M, Lorca J. An analysis of (the lack of) slip transfer between near-cube oriented grains in pure Al. Int J Plast. 2019;118:269-90.

6. Bieler TR, Eisenlohr P, Zhang C, Phukan HJ, Crimp MA. Grain boundaries and interfaces in slip transfer. Curr Opin Solid State Mater Sci. 2014;18:212-26.

7. Bilby A. Types of dislocation sources. In: Bristol conference report on defects in crystalline solids. The Physical Society, London; 1955, p. 124-33.

8. Bayerschen E, McBride AT, Reddy BD, Böhlke T. Review on slip transmission criteria in experiments and crystal plasticity models. J Mater Res. 2016;51:2243-58.

9. Berbenni S, Taupin V, Djaka KS, Fressengeas C. A numerical spectral approach for solving elasto-static field dislocation and g-disclination mechanics. Int J Solids Struct. 2014:51:4157-75.

10. Cahn JW, Mishin Y, Suzuki A. Coupling grain boundary motion to shear deformation. Acta Mater. 2006;54:4953-75.

11. Csiszár G, Misra A, Ungár T. Burgers vector types and the dislocation structures in sputter-deposited Cu-Nb multilayers. Mater Sci Eng A. 2011;528:6887-95.

12. Dewald M, Curtin W. Multiscale modelling of dislocation/grain-boundary interactions: I. Edge dislocations impinging on $\Sigma 11(113)$ tilt boundary in Al. Model Simul Mat Sci Eng. 2007:15:S193-215.

13. Dewald M, Curtin W. Multiscale modelling of dislocation/grain boundary interactions. II. Screw dislocations impinging on tilt boundaries in Al. Philos Mag. 2007;87:4615-41.

14. Dewald M, Curtin W. Multiscale modeling of dislocation/grain-boundary interactions: III. $60^{\circ}$ dislocations impinging on $\Sigma 3, \Sigma 9$ and $\Sigma 11$ tilt boundaries in Al. Model Simul Mat Sci Eng. 2011;19:055002.

15. Djaka KS, Taupin V, Berbenni S, Fressengeas C. A numerical spectral approach to solve the dislocation density transport equation. Model Simul Mat Sci Eng. 2015;23:065008

16. Djaka KS, Villani A, Taupin V, Capolungo L, Berbenni S. Field dislocation mechanics for heterogeneous elastic materials: a numerical spectral approach. Comput Methods Appl Mech Eng. 2017;315:921-42.

17. Fressengeas C, Beausir B. Tangential continuity of the curvature tensor at grain boundaries underpins disclination density determination from spatially mapped orientation data. Int J Solids Struct. 2019;156-157:210-5.

18. Farkas D, Froseth A, Van Swigenhoven H. Grain boundary migration during room temperature deformation of nanocrystalline Ni. Scripta Mater. 2006;55:695-8.

19. Frank FC. The resultant content of dislocations in an arbitrary intercrystalline boundary. In: Symposium on the plastic deformation of crystalline solids. Mellon Institute, Pittsburgh, (NAVEXOS-P-834); 1950, p. 150-4.

20. Fressengeas $C$, Taupin V, Upadhyay M, Capolungo L. Tangential continuity of elastic/plastic curvature and strain at interfaces. Int J Solids Struct. 2012;49:2660-7.

21. Gurtin ME. A theory of grain boundaries that accounts automatically for grain misorientation and grain-boundary orientation. J Mech Phys Solids. 2008;56:640-62.

22. Hadamard J. Leçons sur la propagation des ondes et les équations de l'hydrodynamique. Paris: Herman; 1903.

23. Hall EO. The deformation and ageing of mild steel: III discussion of results. Proc Phys Soc Lond. 1951;64:747-53. 
24. Hémery S, Nizou P, Villechaise P. In situ SEM investigation of slip transfer in Ti-6Al-4V: effect of applied stress. Mater Sci Eng A. 2018;709:277-84.

25. Jiang B. The least-squares finite element method. In: Theory and computation in fluid dynamics and electromagnetics, Springer series in scientific computation. Springer Verlag, Berlin, Heidelberg, New York; 1998.

26. Kröner E. Kontinuumstheorie der Versetzungen und Eigenspannungen. In: Collatz L, Lösch F, editors. Ergebnisse der Angewandte Mathematik, vol. 5. Heidelberg: Springer; 1958. p. 1-179.

27. Kröner E. Continuum theory of defects. In: Balian R, editor. Les Houches, Session XXXV, physics of defects. Amsterdam: North-Holland; 1981. p. 217-314.

28. Kacher J, Robertson IM. In situ and tomographic analysis of dislocation/grain boundary interactions in $\alpha$-titanium. Philos Mag. 2014;94:814-29.

29. Livingston JD, Chalmers B. Multiple slip in bicrystals deformation. Acta Metal. 1957;5:322-7.

30. Li JCM. Disclination model of high angle grain boundaries. Surf Sci. 1972;31:12-26.

31. Luster J, Morris MA. Compatibility of dependence on deformation in two-phase Ti-Al alloys: microstructure and orientation relationships. Metal Mater Trans A. 1995;26:1745-56.

32. Lim LC, Raj M. Continuity of slip screw and mixed crystal dislocations across bicrystals of nickel at $573 \mathrm{~K}$. Acta Metal. 1985;33:183-1577.

33. Lee TC, Robertson IM, Birnbaum HK. TEM in situ deformation study of the interaction of lattice dislocations with grain boundaries in metals. Philos Mag A. 1990;62:131-53.

34. Mach J, Beaudoin AJ, Acharya A. Continuity in the plastic strain rate and its influence on texture evolution. J Mech Phys Solids. 2010;58:105-28.

35. Nye JF. Some geometrical relations in dislocated crystals. Acta Metal. 1953;1:153-62.

36. Priester L, Ballufi RW. Technique for studying the interaction of lattice dislocations with grain boundaries during plastic deformation. J Microsc Spectrosc Electron. 1979;4:615-22.

37. Puri S, Das A, Acharya A. Mechanical response of multicrystalline thin films in mesoscale field dislocation mechanics. J Mech Phys Solids. 2011;59:2400-17.

38. Petch NJ. The cleavage strength of polycrystals. J Iron Steel Inst London. 1953;173:25-8.

39. Priester L. Grain boundaries from theory to engineering. In: Series in materials science 172. Springer Verlag, Heidelberg; 2013.

40. Roy A, Acharya A. Finite element approximation of field dislocation mechanics. J Mech Phys Solids. 2005;53:143-70.

41. Roy A, Acharya A. Size effects and idealized dislocation microstructure at small scales: predictions of a Phenomenological model of Mesoscopic Field Dislocation Mechanics: part II. J Mech Phys Solids. 2006;54:1711-43.

42. Rovinelli A, Proudhon H, Lebensohn RA, Sangid MD. Assessing the reliability of fast Fourier transform-based crystal plasticity simulations of a polycrystalline material near a crack tip. Int I Solids Struct. 2020;184:153-6.

43. Richeton T, Wang GF, Fressengeas C. Continuity constraints at interfaces and their consequences on the work hardening of metal-matrix composites. J Mech Phys Solids. 2011;59(10):2023-43.

44. Spearot DE, Sangid MD. Insights on slip transmission at grain boundaries from atomistic simulations. Curr Opin Solid State Mater Sci. 2014;18:188-95.

45. Shen Z, Wagoner RH, Clark WAT. Dislocation pile-up and grain boundary interactions in 304 stainless steel. Scripta Metal. 1986;20:921-6.

46. Taupin V, Capolungo L, Fressengeas C, Upadhyay M, Beausir B. A mesoscopic theory of dislocation and disclination fields for grain boundary-mediated crystal plasticity. Int J Solids Struct. 2015;71:277-90.

47. Taupin V, Chevy J, Fressengeas C. Effects of grain-to-grain interactions on shear strain localization in Al-Cu-Li rolled sheets. Int J Solids Struct. 2016;99:71-81.

48. Tucker GJ, Zimmerman JA, McDowell DL. Shear deformation kinematics of bicrystalline grain boundaries in atomistic simulations. Model Simul Mat Sci Eng. 2010;18:015002.

49. Upadhyay MV, Capolungo L, Taupin V, Fressengeas C, Lebensohn RA. A higher order elasto-viscoplastic model using Fast Fourier Transforms: effects of lattice curvatures on mechanical response of nanocrystalline metals. Int J Plast. 2016;83:126-52.

50. Varadhan S, Beaudoin AJ, Acharya A, Fressengeas C. Dislocation transport using an explicit Galerkin/least-squares formulation. Model Simul Mat Sci Eng. 2006;14:1245-70.

\section{Publisher's Note}

Springer Nature remains neutral with regard to jurisdictional claims in published maps and institutional affiliations. 